\title{
On a Supposed Puzzle Concerning Modality and Existence
}

\author{
Thomas Atkinson, $^{*} \quad$ Daniel J. Hill ${ }^{\dagger}$ and Stephen K. McLeod ${ }^{\ddagger}$
}

Received: 04 October 2018 / Accepted: 09 February 2019

Abstract: Kit Fine has proposed a new solution to what he calls 'a familiar puzzle' concerning modality and existence. The puzzle concerns the argument from the alleged truths 'It is necessary that Socrates is a man' and 'It is possible that Socrates does not exist' to the apparent falsehood 'It is possible that Socrates is a man and does not exist'. We discuss in detail Fine's setting up of the 'puzzle' and his rejection, with which we concur, of two mooted solutions to it. (One of these uses standard, Kripkean, notions, and the other rests on work done by Arthur Prior.) We set out, and reject, the philosophy of modality underlying Fine's new solution, and we defend an alternative response to the alleged puzzle. Our solution follows the work of David Wiggins in distinguishing between the sentential operator 'It is necessary that' and the predicate modifier 'necessarily'. We briefly provide this distinction with a possibleworld semantics on which it is neither a necessary truth, in some sense, that Socrates exists nor true, in some sense, that Socrates necessarily exists.

\footnotetext{
*University of Liverpool

\thom_atk@me.com

$\dagger$ University of Liverpool

Corresponding author. Department of Philosophy, University of Liverpool, Mulberry Court, Mulberry Street, Liverpool, L69 7ZY, The United Kingdom

\djhill@liverpool.ac.uk

$\ddagger$ University of Liverpool

\skmcleod@liverpool.ac.uk
}

(C) The Authors. Journal compilation (C) The Editorial Board, Organon F. 
Keywords: contingent existents; de re/de dicto distinction; de re modality; existence; Kit Fine; necessity

... if tense logic is haunted by the myth that whatever exists at any time exists at all times, ordinary modal logic is haunted by the myth that whatever exists exists necessarily. (Prior 1957, 48)

\section{A 'puzzle of possible non-existence'}

(Fine 2005, 328) sets out 'a familiar puzzle concerning possible non-existence ... by means of the following argument':

(1) It is necessary that Socrates is a man. ${ }^{1}$

(2) It is possible that Socrates does not exist.

(3) Therefore it is possible that Socrates is a man and does not exist.

Now (Fine 2005, 329) remarks that 'the argument appears to be sound ... yet its conclusion is unacceptable'. According to (Fine 2005, 329) the argument has the following valid logical form: $\square p, \diamond q: \diamond(p \& q)$.

\section{Two mooted, but deficient, responses}

(Fine 2005, 329) rejects 'two responses to the puzzle that are implicit in the literature'. The first response, 'which', according to (Fine 2005, 329), 'derives from the framework of Prior's system Q', employs a modal logic with truthvalue gaps. (Fine 2005, 329) continues that by its lights 'any proposition

\footnotetext{
${ }^{1}$ The sortal involved in the predicate 'is a man' is, in one sense (namely that in which a boy is not a man), a phased sortal rather than a substance sortal. (On the distinction, see, e.g., Wiggins 2001, 30.) In his discussion, however, Fine is concerned with the first sense of the noun 'man' listed in the Oxford English Dictionary: 'A human being, irrespective of age or sex'. In order to engage with his discussion without tampering with his terminology, we follow him in this. All uses of 'man' are to be taken to involve a substance sortal, rather than a phased one. Also, unlike Fine, when we evaluate the truth values of statements about Socrates, we will suppose (in order to avoid unnecessary complexities and philosophical commitments) that the time of evaluation is one at which Socrates is alive.
}

Organon F 26 (3) 2019: 446-473 
concerning an object is taken to be neither true nor false in any world in which the object does not exist'. 2 This gives rise to two notions of necessity. Where $A$ is any truth-bearer, it is weakly necessary that $A$ if and only if there is no possible world at which $A$ is false. It is strongly necessary that $A$ if and only if $A$ is true at every possible world. ${ }^{3}$ The Priorian response puts this distinction to use as follows. The argument from (1) and (2) to (3) is valid so long as the modalities are read weakly throughout. When we convince ourselves that (1) and (2) are true and that (3) is nevertheless false, this is because we slide from a weak reading of the modalities in (1) and (2) to a strong reading in (3). Read that way, the argument is invalid.

The second response, which (Fine 2005, passim) calls 'standard', and which might equally well be called 'Kripkean', invokes a distinction between 'qualified' and 'unqualified' necessity. (See Kripke 2011, 3, on weak necessity.) A statement of the form ' $F a$ ' is unqualifiedly necessary if and only if it is true in all possible worlds. ${ }^{4}$ It is qualifiedly necessary that $F a$ if and only if it is unqualifiedly necessary that if $a$ exists, then $F a$. This is applied to the argument from (1) and (2) to (3) as follows. The necessity involved in (1) is qualified. The possibility involved in (2) is unqualified. (3) is then invalidly concluded, with the sense of possibility involved in (3) being the unqualified sense. For this conclusion to follow, the sense of necessity involved in (1) would need to be the unqualified sense, which it cannot be if it is not necessary (in the unqualified sense) that Socrates exists. It is held that it is not an unqualified necessity that Socrates exists.

We agree with (Fine 2005, 330-4) that both the Priorian and the standard responses to the 'puzzle' are inadequate, not only as responses to the 'puzzle' but as philosophies of modality. Crucially, we agree with Fine that both responses are unable to deny 'that someone who accepts the necessity that Socrates is a man is thereby committed to accepting the necessity that Socrates exists' (Fine 2005, 331). We take it that this is a problem for the Priorian and standard accounts because it means that they fail to deliver on

\footnotetext{
${ }^{2}$ In order to avoid commitment to propositions, this could be reformulated as follows: a statement is neither true nor false if it contains an empty name.

${ }^{3}$ Appeal to possible worlds is not essential here. These notions can be reformulated as follows. It is weakly necessary that $A$ if and only if it is absolutely impossible that $A$ is false. It is strongly necessary that $A$ if and only if it is absolutely impossible that $A$ is not true. If there are truth-value gaps, then weak necessity so formulated is entailed by, but does not entail, strong necessity so formulated. The definitions of modal notions here are not, of course, reductive. It is questionable whether the appeal to possible worlds really has an advantage, in this respect, over a position that views modality as primitive.

${ }^{4}$ Fine formulates this in terms of propositions, not statements.
} 
their philosophical desideratum that it is in no sense true that "whatever exists exists necessarily'. (In the case of the standard account, see further McLeod 2008.)

Let us show why Fine's critique of the Priorian and standard responses to the 'puzzle' is correct.

Let ' $E$ ' be an existence predicate, ' $\square_{W}$ ' be an operator for Priorian weak necessity, ' $\square_{S}$ ' be an operator for Priorian strong necessity, ' $\square_{Q}$ ' be an operator for standard qualified necessity and ' $\square_{U}$ ' be an operator for standard unqualified necessity.

Let us first demonstrate the problem for the Priorian approach. Suppose that $\square_{W} F a \& \neg \square_{S} F a$. It follows from the latter conjunct that there is a possible world at which it is not true that $F a$. It follows from the first conjunct that there is no possible world at which it is false that $F a$. From these two consequences of the conjunction, it follows that there is a possible world at which it is neither true nor false that $F a$. At that world, it is neither true nor false that $E a$. So, it is not the case that $\square_{S} E a$. On the other hand, it follows that $\square_{W} E a$. This is because there is no world at which it is false that $E a$, since the truth or falsehood of a statement at a world depends upon the nonemptiness, in that world, of the singular terms it contains. On the Priorian approach, then, it is necessary that $E a$ in just the same sense in which it is necessary that $F a$. This is a problem for the Priorian approach because, as is clear from the words of Prior used as this article's epigraph, Prior intended to reject the idea that there is a sense in which concrete objects exist necessarily.

The standard approach faces the same difficulty. Suppose that $\square_{Q} F a$ \& $\neg \square_{U} F a$. It follows from the latter conjunct that there is a possible world at which it is false that $F a$. It follows from the first conjunct, by the definition of qualified necessity, that there is no possible world at which it is false that $\neg E a \vee F a$. From these two consequences of the conjunction, it follows that there is a possible world at which it is false that $E a$. So, it is not the case that $\square_{U} E a$. On the other hand, it follows that $\square_{Q} E a$. This is because there is no world at which it is false that $\neg E a \vee E a$. On the standard approach, then, it is necessary that $E a$ in just the same sense in which it is necessary that $F a$. At least for Kripke, the originator of the standard approach, this is a problematic result. In a piece invoking weak necessity, (Kripke 2011, 15, note 11), Kripke is evidently committed to rejecting the idea that there is any sense in which it may correctly be said that whatever exists exists necessarily. 


\section{Fine's response}

\subsection{Matters worldly and unworldly}

(Fine 2005, 321) contends that there is a distinction, analogous to 'a distinction between tensed and tenseless sentences', between worldly and unworldly sentences. On the back of this distinction, Fine alleges that the sorts of truths normally (and properly) regarded as necessary branch into two mutually exclusive kinds: 'the necessary truths proper .... and the transcendent truths' (Fine 2005, 321). Moreover, there are necessary existents, some of which are 'necessary existents proper' and others of which are 'transcendental existents' (Fine 2005, 321).

The suggestion is not that there are transcendent truths and transcendental existents over and above the necessary truths and necessary existents alleged to exist by some necessity-friendly metaphysicians. Rather, it is that necessity-friendly metaphysics usually overlooks some subtleties that are useful (and perhaps even required) for solving modal/existential puzzles and that are independently plausible (Fine 2005, 322, 328).

Let us set out Fine's distinctions and then turn to his attempt to apply them to the supposed modal/existential puzzle.

A truth-apt sentence that is not what Fine calls a 'hybrid sentence' is tensed if and only if 'it can properly be said to be true or false at a time' (Fine 2005, 322). Otherwise, it is tenseless. Fine's examples of tenseless sentences (Fine 2005, 322) are 'Socrates is a man' and 'Socrates is self-identical'; his example of a tensed sentence is 'Socrates does not exist'. The predicates 'is a man' and 'is self-identical' are in turn classified as tenseless, 'while a predicate such as "exists" is tensed' (Fine 2005, 322).

Now, says Fine, given that we have the distinction between tensed and tenseless sentences, and so between tensed and tenseless truths, we can make 'a corresponding distinction between sempiternal and eternal truths, a sempiternal truth being a tensed sentence that is always true and an eternal truth being a tenseless sentence that is true simpliciter.' In 'a restricted sense of truth-at-a-time' (Fine 2005, 323), only sentences true at some times and false at others, plus sempiternal truths, are true-at-a-time. In 'an extended sense of truth-at-a-time' (Fine 2005, 323) tenseless truths can be taken to be true at all times. The tenseless truths then qualify as being true-at-a-time, since 
truth at all times entails, given that there are times, truth at some times. On the extended conception of truth-at-a-time, 'an eternal truth will be true regardless of the time, i.e. regardless of how things are at the time, while a sempiternal truth will be [true] whatever the time, i.e. however things are at the time' (Fine 2005, 323). By way of examples, (Fine 2005, 323-4) remarks that "If the sun will always shine, then "the sun will shine" will be true at any given time because of how things are at that time ... while the ... truth of "Socrates is [self-]identical" will not depend ... upon some ongoing feature of the universe'.

Let us now move from the tensed/tenseless distinction to the distinction Fine wishes to draw, by analogy with it, between worldly and unworldly matters. A truth-apt sentence that is not one of Fine's 'hybrid sentences' is worldly if and only if it depends for its truth-value 'upon the worldly circumstances' (Fine 2005, 321). Otherwise, it is unworldly. Among the truths that Fine takes to be normally and rightly regarded as necessary by philosophers, the necessary truths are true whatever the worldly circumstances, whereas the transcendental truths are true regardless of the worldly circumstances (Fine 2005, 324). Fine provides 'Socrates is self-identical' as an example of a truth that is unworldly and transcendental. ${ }^{5}$ Earlier, it was cited by him as an example of a tenseless truth. In contrast with transcendental truths, necessary truths like 'Socrates exists or does not exist' depend upon the worldly circumstances. The example is a disjunction, and a disjunction depends for its truth on the worldly circumstances if and only if each of the disjuncts depends for its truth on the worldly circumstance (Fine 2005, 324, 326); each of 'Socrates exists' and 'Socrates does not exist' is a purely worldly sentence. Fine is supposing that if a set $S$ contains only worldly sentences, then any compound formed only from elements of $S$ and truth-functional operators is also a worldly sentence. This entails that a worldly atomic sentence makes any truth-functional compound containing finitely many instances of that sentence and no other atomic sentences worldly too.

Fine's next step is to propound an 'extended' sense of truth-at-a-world to parallel his earlier 'extended' sense of truth-at-a-time. Just as there he had taken the tenseless truths to be true at all times, under the extended notion of truth-at-a-time, so now he takes the transcendental truths to be true at all possible worlds, under the extended notion of truth-at-a-world. In

${ }^{5}$ For Fine, the notions of transcendental truth and of purely unworldly truth are co-extensive.

Organon F 26 (3) 2019: 446-473 
the extended sense of 'necessary' the transcendental truths may be said to be necessary, even though in the restricted sense of 'necessary' Fine distinguishes between the transcendental truths and the necessary truths proper. There is, however, a further extension here whose application to temporality is not canvassed by Fine, and that is the 'superextended' sense of truth-at-a-world. This applies to 'all propositions whatever' (Fine 2005, 326); thus, it applies not just to the worldly sentences and the unworldly sentences, but also to hybrid sentences, that is sentences 'that are composed of both worldly and unworldly components' (Fine 2005, 326). An example would be 'Socrates does not exist or is self-identical'. This sentence is a hybrid sentence, since it is a disjunction of a worldly sentence and an unworldly sentence, and is necessary in the super-extended sense, but not in the extended sense or the restricted sense. ${ }^{6}$

\subsection{Fine's application of the worldly/unworldly distinction to the 'puzzle'}

Recall that the apparent puzzle arises out of the following argument:

(1) It is necessary that Socrates is a man.

(2) It is possible that Socrates does not exist.

(3) Therefore it is possible that Socrates is a man and does not exist.

The supposed puzzle consists, in the words of (Fine 2005, 329), in the fact that 'the argument appears to be sound ... yet its conclusion is unacceptable'. For Fine, solving the 'puzzle' consists not only in pronouncing on whether the argument really is sound or not, but also in explaining why our intuitions are pulled in different ways. So, how are Fine's distinctions meant to help solve the supposed puzzle?

Fine suggests two hypotheses that are fundamental for his solution: (i) the hypothesis that "we are naturally inclined to use the modalities in an

\footnotetext{
${ }^{6}$ For more details about Fine's conceptions of restricted, extended, and super-extended modalities, see the Appendix to this article.
} 
unextended or extended sense, though not in a superextended sense' (Fine 2005, 335); (ii) the hypothesis that "exists" is a worldly predicate while such predicates as "man" or "identity" are unworldly' (Fine 2005, 336). So, says (Fine 2005, 336),

$[\mathrm{w}]$ hen it comes to evaluating the first premiss [...], we implicitly treat 'man' as an unworldly predicate (in conformity with the [second] hypothesis). We then accommodate the sense of 'necessity' to the sense of 'man' by taking it in an extended sense (by the first hypothesis) and are thereby led to accept the first premiss.

Moreover, Fine thinks we are correct so to do, since he thinks 'is a man' is an unworldly predicate, and so 'Socrates is a man', since it is true, is a transcendental truth, and therefore an extended necessity. In evaluating the second premise, "we implicitly treat "exists" as a worldly predicate (in conformity with the [second] hypothesis). We then take "possibility" in the unextended sense (by the first hypothesis) and are thereby led to accept the second premiss' (Fine 2005, 337). Again, we are correct so to do, since 'exists' is, according to Fine, in this context, a worldly predicate, and so, given that, intuitively, it is possible that Socrates does not exist, the sense of 'possible' here must be the restricted or unextended sense.

Now, for the dénouement. By Fine's own earlier definitions, the right thing for us to do is to take 'Socrates is a man and does not exist' to be a hybrid sentence, since 'is a man' is a transcendental predicate and 'does not exist' is a worldly one. On this account the right way for us to understand the use of 'possible' in (3) would then be as a super-extended use, and (3) would then come out as true. Fine has to explain why we intuitively think that (3) is false. He says that this is because by his first hypothesis we are 'disinclined' to use the super-extended sense of 'possible'; when we evaluate the conclusion we now take 'is a man' to be worldly (and so 'possible' in the restricted or unextended sense), contrary to how we took it in the first premiss. Fine explains that 'under this option, we take the possibility that Socrates is a man and does not exist to consist in the existence of a possible worldly circumstance in which Socrates is a man and does not exist' (Fine 2005, 337), and it is this that we rightly reject. Why do we (wrongly, by Fine's lights) take it to consist in the existence of that worldly circumstance? Fine seems to think that we are distracted by the presence of the word 'exist' in (3) into

Organon F 26 (3) 2019: 446-473 
confusing the transcendental predicate 'is a man' with the worldly predicate 'is an existent man'. (To those worried that 'is an existent man' ought to be a hybrid predicate rather than a worldly one, Fine replies 'we may use the worldly predicate ["existent"] to qualify the unworldly predicate ["is a man"] $[\ldots]$ and thereby form a worldly predicate [that] is true in each world of those objects that are in the world-free extension of the first predicate and in the world-bound extension of the second predicate' (Fine 2005, 337).) On our incorrect, worldly, understanding of 'is a man' the premisses do not entail the conclusion, since the argument is now of the invalid form: $\square_{T} p, \diamond_{R} q: \diamond_{R}(p$ $\& q)$.

On the correct, unworldly, understanding of 'is a man', however, since 'Socrates is a man and does not exist' is a hybrid sentence, the sense of 'possible' involved is the super-extended sense. Since Fine thinks that we are already committed to the interpretation that the conclusion uses the superextended sense of 'possible', he suggests that we should 'use the modalities in a superextended sense throughout the argument', in which case 'the conclusion does indeed follow from the premisses and should therefore be accepted' (Fine 2005, 338). On this hypothesis the argument contains no false premiss and is of the valid form $\square_{S E} p, \diamond_{S E} q: \diamond_{S E}(p \& q)$.

Having set out the alleged puzzle, two deficient responses to it and Fine's own response, in the following two sections we turn to the evaluation of Fine's response and present our own response.

\section{Dissolving the 'puzzle'}

We hold, contrary to (Fine 2005, 328), that it is not the case that the 'puzzle' concerning possibility and non-existence 'would otherwise be quite baffling' without our having Fine's worldly/unworldly distinction at our disposal to help us solve it. (Fine 2005, 328) holds that the application of the worldly/unworldly distinction to solve the 'puzzle' counts as 'an important point in its favour'. While we do not argue against it here, the worldly/unworldly distinction appears to us, like Fine's version of the tensed/tenseless distinction, to be contentious. In fact, although we do not develop this point in what follows, we doubt that Fine has succeeded in articulating any genuine distinction between matters worldly and unworldly. We will now argue in favour of 
what we think is a better approach to the supposed puzzle than those we have discussed so far.

On our account, the problem with the argument from (1)-(3) is a lot simpler than the switch between different understandings of necessity put forward by the responses discussed so far (Priorian, standard and Finean). ${ }^{7}$

We begin by distinguishing, after (Wiggins 2001, 111-4), between two sorts of sentence that feature in natural language: statements of necessary truth, like

(1) It is necessary that Socrates is a man.

and statements of de re necessity like

$\left(1^{*}\right)$ Socrates is necessarily a man.

The modal operator in (1) is an operator on sentences. That in $\left(1^{*}\right)$ is embedded within a complex predicative expression and modifies a simpler predicative expression. We assume, after Wiggins, that statements of de re necessity are not generally reducible to statements of necessary truth. We also assume, again after Wiggins, that a true statement of de re necessity, when it is about a contingent being and contains no operator other than the modal operator, differs in truth value from its de dicto counterpart (which is, in virtue of the contingency of the contingent being's existence, false).

According to this approach, modal logics that employ only sentential modal operators cannot therefore adequately capture the distinction between modality de dicto and modality de re (cf. Wiggins 1976, 294). In order to illustrate this distinction formally, Wiggins employs the device of lambda abstraction, which is designed to create a predicate into which predicate modifiers can easily be inserted, and the predicate-modifier ' $\mathrm{NEC}$ '.

(1) It is necessary that Socrates is a man.

$$
\square[(\lambda x)[\operatorname{Man} x],\langle\text { Socrates }\rangle]
$$

\footnotetext{
${ }^{7}$ Since (Fine 2005, 338) ultimately deems the argument sound, he thinks that the problem mainly lies not in the argument itself but in our intuitive analysis of it. This marks a difference between his approach, on the one hand, and, on the other, the Priorian approach, the standard approach and our own.

${ }^{8}$ While it is convenient to Wiggins's discussions and to ours to describe de re 'necessarily', and 'NEC', as predicate-modifiers, (Wiggins 2001, 111) expresses neutrality over whether these expressions really modify predicates or rather the copula. For a discussion of these options, and a defence of the view that modal adverbs are copula modifiers, see (McGinn 2000, Chapter 4).
}

Organon F 26 (3) 2019: 446-473 
$\left(1^{*}\right)$ Socrates is necessarily a man.

$[\operatorname{NEC}(\lambda x)[\operatorname{Man} x]],\langle$ Socrates $\rangle$

Approaches to the de re/de dicto distinction that employ only sentential modal operators will have difficulty in justifiably securing the result that in statements of necessity de re, like $\left(1^{*}\right)$, the name does not occur in an opaque context (again, cf. Wiggins 1976, 294). It is therein, rather than in considerations about the scope of a sentential modal operator (which is thereby, at least as normally understood in the literature, an intensional, opacity-producing operator), that the distinction between de re and de dicto modal statements is genuinely to be identified.

Let us assume, with Fine, and as the surface grammar of its constituent sentences suggests, that the argument (1)-(3) employs only sentential modal operators.

We contend, contrary to (1), that it is not, in fact, necessary that Socrates is a man. We think this because, intuitively, it might have been the case that there never was such an object as the concretely existent philosopher Socrates, and, as (Rumfitt 2003, 466) puts it, 'an object's existence is a pre-condition for its being either true or false that the object is such-and-such'. (Rumfitt clearly means that it is a necessary pre-condition not a merely contingent one.) We know that Fine disputes Rumfitt's and our intuitions here, but we are not persuaded by his attempts (Fine 2005, 345) to explain how something can be a man without existing.

If, however, we are wrong about this, and (1) is correct, and it is indeed necessary that Socrates is a man for the reason that Fine gives, viz. that it is a transcendental truth that he is a man, then we affirm that in that case (2) is false. If it is transcendentally true that Socrates is a man then Socrates will enjoy, along with his worldly existence, an unworldly, transcendental form of existence, and, since this is transcendental, it will be impossible that he should not have enjoyed it. We know that Fine will reply that, although (2) can legitimately be taken as coming out false in this way, it is more natural to interpret it as being about worldly existence and therefore to take it to be true. All we can say is that we find implausible the proposed switch from transcendental necessity in (1) to worldly necessity in (2), and that strong reasons would be required to overcome this implausibility. Moreover, if we are right that the approach to modality that we propose disposes of the supposed puzzle, then the strong reasons to adopt Fine's switch of modalities between 
the first and the second premiss are lacking.

As we remarked in Section 3.2, Fine thinks that solving the 'puzzle' consists partly in evaluating the soundness of the argument and partly in explaining why our intuitions are pulled in different ways when we try to evaluate its soundness. ${ }^{9}$ Fine presented the argument as one in which we have two intuitively true premisses which, by a valid rule of inference, entail a conclusion that strikes us as intuitively unacceptable.

We can explain the intuition that (1) is true by saying that, when (1) is (mistakenly) evaluated as true, it is being taken to be a notational variant of $\left(1^{*}\right)$. We take it that if sortal essentialism is a true metaphysical doctrine and 'man' is a substance sortal, then 'Socrates is necessarily a man' is indeed true. Sortal essentialism, does not, we have agreed with Wiggins, entail (1) itself.

Regarding (2), if both the modality and the negation are taken to be sentential, then the original supposed intuition that (2) is true can be left intact. After all, we have argued that the embedded statement in (1) (i.e. the statement 'Socrates is a man') is in no genuine sense alethically necessary and it follows from this, given its truth-aptness, that there is some sense in which its negation is alethically possible. Reluctance to accept (3) is, we have argued, entirely well-founded. Even if it is held, for the reasons we have just mentioned, that (1) and (2) are intuitively true, the attendant reluctance, nonetheless, to accept (3) does not require the employment of Fine's distinction between matters worldly and unworldly (and the distinctions he launches on its back).

We can now set up a dilemma concerning the interpretation of (1):

(1) It is necessary that Socrates is a man.

There are two possible interpretations of (1). The first is that, in line with its surface syntax, it is a statement that employs a sentential modal operator:

$$
\square[(\lambda x)[\operatorname{Man} x],\langle\text { Socrates }\rangle]
$$

The second is that it is to be interpreted as a notational variant of $\left(1^{*}\right)$ ('Socrates is necessarily a man'), which we formalized as:

$$
[\operatorname{NEC}(\lambda x)[\operatorname{Man} x]],\langle\text { Socrates }\rangle
$$

\footnotetext{
${ }^{9}$ In discussion, Fine has emphasized to us the importance, in his estimation of the philosopher's remit, of the second part of this task.
}

Organon F 26 (3) 2019: 446-473 
Assume, for the first horn of the dilemma, the first interpretation, i.e., that (1) employs a sentential modal operator. In that case, as we suggested above (after Rumfitt), (1) entails

(4) It is necessary that Socrates exists.

$\square[(\lambda x)[$ Exists $x],<$ Socrates $>]$

Lest this formalization should give the impression that we admit a primitive first-level existence predicate, we now provide what we take to be a notational variant of it:

$$
\square[(\lambda x)[(\exists y) y=x],<\text { Socrates }>]
$$

This variant employs the well-known classical-logical device of using the existential quantifier and the identity sign to formalize singular existentials. ${ }^{10}$ Throughout, when we use 'Exists' in lambda translations, we intend to employ it merely as an abbreviation, not as a primitive first-level predicate.

Let us return to the main business. Provided that the modalities involved in (1) and (2) are duals and that (2) also employs a sentential modal operator, and given that (1) entails (4), (1) and (2) form an inconsistent pair. Thus, no puzzle is generated: the argument is unsound since one of the premises is false.

Assume, for the second horn of the dilemma, that (1) is to be interpreted as a notational variant of $\left(1^{*}\right)$. As a result, the argument is no longer of the valid form $\square p, \diamond q: \diamond(p \& q)$. Instead, the argument is now:

$\left(1^{*}\right)$ Socrates is necessarily a man.

$[\operatorname{NEC}(\lambda x)[\operatorname{Man} x]],\langle$ Socrates $\rangle$

(2) It is possible that Socrates does not exist.

$$
\diamond \neg[(\lambda x)[\text { Exists } x],\langle\text { Socrates }\rangle]
$$

\footnotetext{
${ }^{10} \mathrm{~A}$ translation that is more complicated still, though reducible to ours, can be obtained by emulating the approach suggested by (Wiggins 2003, 486). Thus: ' $\square[[(\exists y)((\lambda x=$ Socrates $],\langle y\rangle]$ ' . This illustrates the ability of the lambda notation to reflect the grammatical distinction between 'Socrates is identical with something' (our formulation) and 'Something is identical with Socrates' (Wiggins 2003).
} 
(3) Therefore it is possible that Socrates is a man and does not exist.

$\diamond[(\lambda x)[\operatorname{Man} x],\langle$ Socrates $\rangle \& \neg[(\lambda x)[$ Exists $x],\langle$ Socrates $\rangle]]$

Thus, the argument is invalid, since the two premises employ different modalities: $\left(1^{*}\right)$ uses de re necessity, and does not entail its de dicto counterpart, while (2) uses de dicto possibility. Once again, there is no puzzle.

\section{De re necessity and contingent existence}

Our approach to dissolving the 'puzzle' involved appeal to a version of the distinction between necessity de re and de dicto that remains unorthodox. Relatedly, its technical details remain relatively under-developed. In particular, it might be objected that our approach does not seem to be readily compatible with model-theoretical approaches to the formal semantics of modal logics. Our response to this objection consists of two points. First, we note that the form of distinguishing between the logical syntax of de re and de dicto modal sentences that we have favoured, including the deployment of the lambda notation, has already been adopted by some authors who also, in fact, provide both types of sentence with model-theoretical semantics: (Fitting and Mendelsohn 1998, 85-8, 193, 201, 213, 217) and (Garson 2013, Chapter 19). Secondly, this section and the next one explain the distinction in more detail and summarize a model-theoretical semantics that preserves the judgements made in the foregoing sections about the truth values of relevant modal sentences.

We take it that if Socrates is a contingent being then it is neither necessary that Socrates exists nor the case that Socrates necessarily exists. Thus, we take both of the following sentences to be false:

(4) It is necessary that Socrates exists.

$\square[(\lambda x)[$ Exists $x],\langle$ Socrates $\rangle]$

$\left(4^{*}\right)$ Socrates necessarily exists.

On the assumption that there is no primitive first-level existence predicate, and that 'Socrates exists' contains neither the copula nor such a predicate,

Organon F 26 (3) 2019: 446-473 
the 'necessarily' in $\left(4^{*}\right)$ cannot be either a copula modifier or the modifier of such a predicate. On our view, given that Socrates is a contingent existent, $\left(4^{*}\right)$ admits of no true essentialist reading, and is significantly different from standard essentialist statements like $\left(1^{*}\right)$.

We think that someone unacquainted with formal systems of modal logic that took $\left(1^{*}\right)$ to be true would be unlikely to regard $\left(4^{*}\right)$ as true. We think that such a person would understand $\left(4^{*}\right)$ as asserting Socrates to be a necessary being, rather than merely necessarily being a being (or necessarily being an object). We do not intend this to be a merely pragmatic point. Rather, our point is that taking $\left(4^{*}\right)$ to admit of an interpretation on which it is true appears to us to be subversion of what the sentence means. ${ }^{11}$

Someone might offer this formalization of $\left(4^{*}\right)$, treating the sentence in broadly the same manner as $\left(1^{*}\right)$ above:

$$
[\operatorname{NEC}(\lambda x)[(\exists y) y=x]],\langle\text { Socrates }\rangle
$$

It seems to us that this formalization is apt for (the true) 'Socrates is necessarily a being', but is not apt for (the false) 'Socrates is a necessary being' and, thus, not apt for (the false) $\left(4^{*}\right)$ (i.e., 'Socrates necessarily exists').

Our position with respect to (4) and $\left(4^{*}\right)$ just spells out what we think is involved in holding that Socrates is a contingent, rather than (unlike such supposed cases as God, numbers, and propositions) a necessary, being. With respect to $\left(4^{*}\right)$, we agree with (Fine 2005,329$)$ :

There may be items - numbers or propositions or the like - that necessarily exist. But Socrates (and Felix) are not among them. Indeed, it is surely possible that no men (or cats) exist; and from this it follows, given the appropriate version of the first premiss, that it is possible that Socrates (or Felix) does not exist.

In denying that $\left(1^{*}\right)$ entails $\left(4^{*}\right)$, we part company with a philosopher whose general approach to the distinction between modality de dicto and de re we share, namely Efird.

\footnotetext{
${ }^{11}$ Also, when people that think that there are contingent beings suggest that $\left(4^{*}\right)$ is true, we believe that they interpret $\left(4^{*}\right)$ in a way that dilutes its real-and, in philosophy and theology, traditional-meaning. When theologians say that God necessarily exists they are not appealing to a notion of necessary existence that is true of all objects. Indeed, we deny that there is any such bona fide notion. The suggestion that there is some sense of 'necessarily' on which $\left(4^{*}\right)$ is true seems to us to stem either from the global denial of contingent existence or from a reconstruction of natural-language meaning in the light of the features of a formal system of modal logic.
} 
Efird's approach combines a Wiggins-style approach to the de re/de dicto distinction with a Priorian semantics. It has the result (Efird 2010, 107), as we also contend, that there are no necessary truths about contingent existents. Thus, to use Efird's example, it is not the case that it is necessary that Timothy Williamson exists. Nevertheless, Efird's approach has the further result (Efird 2010, 97, 107), which ours does not, that Williamson necessarily exists. ${ }^{12}$ According to (Efird 2010, 107), when the Priorian semantics is adopted: ${ }^{13}$

we can $[. .$.$] define a scheme for translating formulas containing$ 'nec' to sentences containing ' $\diamond$ ' and ' $\neg$ ': $[\operatorname{nec}(\lambda x)(P(x))],[a]={ }_{d f}$. $\neg \diamond \neg P(a)$. This then yields the following [...]:

'Necessarily, $a$ is $\mathrm{F}$ ' is represented formally as: $\square \mathrm{F} a$.

' $a$ is necessarily $\mathrm{F}$ ' is represented formally as: ( $\mathrm{F} a$ \& $\neg \diamond \neg \mathrm{Fa}$ ).

'Contingently, $a$ is $\mathrm{F}$ ' is represented formally as: $(\mathrm{F} a \&$ $\neg \square \mathrm{F} a)$.

' $a$ is contingently $\mathrm{F}$ ' is represented formally as: $(\mathrm{F} a$ \& $\diamond \neg \mathrm{F} a)$.

These formalizations give rise to the consistent conjunctive schema:

' $a$ is necessarily $\mathrm{F}$ and, contingently, $a$ is $\mathrm{F}$ ' is represented formally as: $[\mathrm{F} a \&(\neg \diamond \neg \mathrm{F} a \& \neg \square \mathrm{F} a)]$.

As an instance of this conjunctive schema we have: Williamson necessarily exists, and, contingently, Williamson exists $[\exists ! a \&(\neg \diamond \neg \exists ! a$ $\& \neg \square \exists ! a)]$. So [...] Williamson is, in a de re sense, a necessary existent, but he is not a necessary existent in the de dicto sense:

\footnotetext{
${ }^{12}$ We note that (Efird 2010, 106) assumes that Wiggins's account of de re 'necessarily' properly applies to modalized existentials like $\left(4^{*}\right)$, and appears to see no difficulties with this assumption. We have argued, in this section, that it does not so apply, and, in so doing, we have made such difficulties plain. Also, (Wiggins $1995,2003)$ seems to favour treating 'exists', as we do, as a defined predicate. So, the proponent of a Wigginsstyle approach to the de re/de dicto distinction does not seem to be forced to go down Efird's road of taking it that there is a sense in which 'Socrates necessarily exists' is true.

${ }^{13}$ Efird uses 'nec' in place of Wiggins's 'NEC', 'ヨ!' as an existence predicate, and square brackets both where Wiggins would use square brackets and in place of Wiggins's angled brackets.
}

Organon F 26 (3) 2019: 446-473 
Williamson necessarily exists, but it is not necessary that he exists. $^{14}$

Importantly, for our purposes, Efird's approach has the consequence that it is true that Williamson necessarily exists. Efird has to affirm, then, that $\left(1^{*}\right)$ entails $\left(4^{*}\right)$; indeed, he embraces this consequence. On the interpretation of $\left(4^{*}\right)$ that Efird gives, $\left(4^{*}\right)$, though true, does not entail (4), and does not contradict (2). On Efird's account, just as each object is necessarily identical with itself, so each object necessarily exists. Let us set out, and explain the reasoning behind, our own position. Consider the following sentences:

(5) Socrates is necessarily self-identical.

$[\operatorname{NEC}(\lambda x[x=x]],\langle$ Socrates $\rangle$

(6) Socrates is necessarily an existent.

$[\operatorname{NEC}(\lambda x)[$ Existent $x]],\langle\text { Socrates }\rangle^{15}$

(7) Socrates is necessarily an object.

$[\operatorname{NEC}(\lambda x)[$ Object $x]],\langle$ Socrates $\rangle$

(8) Socrates is a necessary existent.

$\square[(\lambda x)[$ Existent $x],<$ Socrates $>]$

Supposing that $\left(1^{*}\right)$ is true, $(5)$ follows, for whatever is necessarily a man is also necessarily self-identical. Likewise, and with similar reasoning, (6) and (7) also follow from $\left(1^{*}\right)$. While it is the case that whatever is necessarily a

\footnotetext{
${ }^{14}$ It is evident, then, that Efird holds that 'Williamson is a necessary existent' is ambiguous. In the main text, we deny that it is ambiguous. Moreover, even if, as Efird argues, 'Williamson necessarily exists, and, contingently, Williamson exists' is true, it does not follow immediately that there are two senses of 'Williamson is a necessary existent'. This is because that sentence employs neither a modal operator nor a modal adverb, but, rather, a modal adjective. We note, further, that non-existential sentences that employ 'necessarily' as a predicate modifier, like 'Socrates is necessarily human', do not seem equivalent to the corresponding sentences featuring the adjective 'necessary', like 'Socrates is a necessary human': it is questionable whether these transformations yield sentences that are well-formed and semantically complete, let alone ones that mean what the source sentences mean. This seems to be a difference between modalized non-existentials and modalized existentials, like 'Socrates necessarily exists', which do seem equivalent to the corresponding sentences featuring the adjective 'necessary' (in this case, 'Socrates is a necessary existent').

${ }^{15}$ In this formalization, 'Existent' is not to be read adjectivally. Compare 'Object' in (7).
} 
man is necessarily an object, and necessarily an existent, it is not the case that whatever is necessarily a man is a necessary object or a necessary existent. Thus (8), unlike (5)-(7), does not follow from $\left(1^{*}\right)$. While (8) entails that it is necessary that there is such an existent as Socrates, (6) and (7) do not.

$\left(4^{*}\right)$ might at first appear to admit of two different readings, namely (6) and (8). Nevertheless, we see no good reason to view (6) as an admissible reading of $\left(4^{*}\right)$. While whatever exists is necessarily a being, an object, or an existent, this does not entail that whatever exists exists necessarily: thus, (6) is logically weaker than $\left(4^{*}\right)$. If, as we believe, $(6)$ is an incorrect reading of $\left(4^{*}\right)$, then $(8)$ is the correct reading. ${ }^{16}$ The distinction between (6) and (8) seems as clear to us as that between the true 'Socrates is necessarily a being' and the false 'Socrates is a necessary being'. Since (8) admits of no ready reading on which it is true, and since (6) is not available as an independently plausible, rather than theoretically forced, reading of $\left(4^{*}\right),\left(4^{*}\right)$ also admits of no ready reading on which it is true.

\section{Semantics for de re necessity with contingent existence}

While it might be thought that a Wiggins-style approach to the syntax of de re modality is not readily reconcilable with the standard model-theoretical (or 'possible worlds') semantics for modal logics, we noted, at the beginning of the previous section, that this is not really the case. What has been lacking in the literature so far, however, is a semantics that secures the intuitive judgements about the truth values of modal sentences that partly motivate the Wigginsstyle approach, and that is a semantics consistent with the judgement that there is no sense of 'necessarily' in which 'whatever exists exists necessarily' is true.

We now specify a semantics fit to accommodate the attribution of de re

\footnotetext{
${ }^{16}$ We take this to be so independently of whether the lambda translation given for (8) is ultimately correct. One reason for doubt here is that in sentences like 'Socrates necessarily exists', the alternatives we have explored leave us with no method of formalization that both captures the logical strength of the sentence and allows 'necessarily' to remain, as it appears, a referentially transparent operator. Also, (8) features not a modal operator or adverb, upon which modal logics have overwhelmingly concentrated, but a modal adjective. The logical behaviour of modal adjectives is neither studied as often nor understood as well. There seems to be work left to do in resolving these difficulties.
}

Organon F 26 (3) 2019: 446-473 
necessary attributes to beings that are held to exist contingently. ${ }^{17}$ Our semantics has the following features.

First, no atomic sentence is a logical truth. In classical quantificational logics, any identity sentence of the form $\ulcorner c=c\urcorner$ is a logical truth. In classicallybased quantified modal logics, any formula of the form $\ulcorner\square c=c\urcorner$ is normally a logical truth. (For an exception, see Nelson 2016.) Since, on our account, no atomic sentence is a logical truth, we avoid the result that, for any arbitrarily selected object, it is a true statement of de dicto necessity that the object is identical with itself.

Secondly, our semantics is that of a universally free negative free logic. That is to say, as well as employing free-logical quantification (Garson 2013, Ch. 13, esp. 264), and permitting both empty names and an empty domain, we treat every atomic sentence containing an empty name as thereby false.

Thirdly, the presence of empty names, does not, on our semantics, give rise to trivially true statements of de re necessity. For example, it is a logical truth, because it is an instance of the law of identity of propositional logic, that if Pegasus exists then Pegasus exists. As a consequence, when the weak necessity account of de re necessity is adopted, and extended to natural language, Pegasus necessarily exists, and, indeed, necessarily - albeit trivially so - has every property whatsoever, as a matter of weak necessity - see further (McLeod 2008, 319).

Fourthly, essentialist facts about contingent beings, if there be such facts, are metaphysical: they are not logical truths. They have the same logical status as atomic facts. Thus, the de re necessitation of an atomic sentence, which involves the insertion of a predicate-modifying 'necessarily' into the sentence (as when 'Socrates is human' is turned into 'Socrates is necessarily human') will have a result that, like the simpler sentence, is neither logically true nor logically false.

We now spell out the semantic differences between, $(1)$ and $\left(1^{*}\right)$; we then provide general semantic clauses for the modal expressions in these sentences. In what follows, following (Garson 2013), 'wRv' means that the possible world $\mathbf{w}$ has access to the possible world $\mathbf{v}$ (in other words, $\mathbf{v}$ is accessible from $\mathbf{w}$ ). ' $\mathbf{a}_{\mathbf{w}}$ (It is necessary that Socrates is a man $)=T$ ', for example, means that the sentence in parentheses is true at the world $\mathbf{w} .^{18}$

\footnotetext{
${ }^{17}$ We do not here attempt fully to specify a formal system fit for this task. We hope to do so elsewhere.

${ }^{18}$ Our semantic clause for 'It is necessary that' mirrors that of ' $\square$ ' in the modal logic K: see (Garson 2013, 64). That for predicate-modifying 'necessarily' is different.
} 
Semantics for (1):

$\mathbf{a}_{\mathbf{w}}$ (It is necessary that Socrates is a man $)=\mathrm{T}$ if and only if, for each possible world, $\mathbf{v}$, such that $\mathbf{w R v}, \mathbf{a}_{\mathbf{v}}$ (Socrates is a $\operatorname{man})=\mathrm{T}$.

Semantics for $\left(1^{*}\right)$ :

$\mathbf{a}_{\mathrm{w}}$ (Socrates is necessarily a man) $=\mathrm{T}$ iff $\mathbf{a}_{\mathrm{w}}$ (Socrates exists $)=\mathrm{T}$, and for each possible world, $\mathbf{v}$, such that $\mathbf{a}_{\mathbf{v}}($ Socrates exists $)=\mathrm{T}$ and $\mathbf{w} \mathbf{R v}, \mathbf{a}_{\mathbf{v}}($ Socrates is a man $)$ $=\mathrm{T}$.

Semantics for 'It is necessary that':

$\mathbf{a}_{\mathbf{w}}$ (It is necessary that $c$ is $\left.A\right)=\mathrm{T}$ iff for each $\mathbf{v}$ such that $\mathbf{w R v}, \mathbf{a}_{\mathbf{v}}(c$ is $A)=\mathrm{T}$.

Now let $A$ be a primitive predicate (that is, one that is not defined using any item, other than the identity sign, from the logical vocabulary). ${ }^{19}$ With this restriction in place, we now specify our semantics for predicate-modifying 'necessarily'.

Semantics for predicate-modifying 'necessarily':

$\mathbf{a}_{\mathbf{w}}(c$ is necessarily $A)=\mathrm{T}$ iff $\mathbf{a}_{\mathbf{w}}(c$ exists $)=\mathrm{T}$ and for each $\mathbf{v}$ such that $\mathbf{a}_{\mathbf{v}}(c$ exists $)=\mathrm{T}$ and $\mathbf{w} \mathbf{R v}, \mathbf{a}_{\mathbf{v}}(c$ is $A)$ $=\mathrm{T}$.

On these semantics, (1), but not $\left(1^{*}\right)$, entails that it is necessary that Socrates exists. Moreover, $\left(1^{*}\right)$ does not entail, in any bona fide sense of 'necessarily', the sentence 'Socrates necessarily exists'. Our semantics for predicatemodifying 'necessarily', though simple, is intended to achieve the breakthrough of providing for a Wiggins-style account of the de re/de dicto distinction a

\footnotetext{
${ }^{19}$ While this restriction may go further than is strictly necessary in that it excludes all defined predicates, it is motivated by the case of 'exists', as discussed in Section 5 above. The discussion there was intended, in part, to show that the exclusion of 'exists', at least, from the clause for predicate-modifying 'necessarily' was not an arbitrary restriction.
}

Organon F 26 (3) 2019: 446-473 
model-theoretical semantics that allows there to be true statements of de re necessity about contingent beings, without having the result that, in some sense of 'necessarily', the statement 'whatever exists exists necessarily' is true.

The defined status of the existence predicate means that 'Socrates necessarily exists', for which our semantic clause for predicate-modifying 'necessarily' does not account, must be parsed in one of the following ways:

(9) It is necessary that there is something with which Socrates is identical.

(10) There is something that is necessarily identical with Socrates.

On the first parsing, which is the one that both natural language and traditional philosophical and theological parlance about necessary beings would suggest to be appropriate, there is (unlike in cases that feature a primitive monadic predicate) no semantic difference between 'Socrates necessarily exists' and 'It is necessary that Socrates exists'. (Given that it is not the case that Socrates exists in all possible worlds, both sentences are false.) Parsed in the second way, 'Socrates necessarily exists' is true, but its surface form very much belies both its logical form and its semantics. Moreover, the proposal that the sentence ought to be so parsed is one for which we see no good rationale and against which we have already argued in Section 5.

\section{Conclusion}

We conclude that the argument from (1)-(3) is unsound, and that it does not generate any deep puzzle. The predominant reason given by Fine for subscribing to the worldly/unworldly distinction is as a means of solving the 'puzzle', with any other reasons being only alluded to, rather than spelled out, in his piece. Thus the distinction is, on the evidence Fine presents, undermotivated. In the case of the supposed puzzle, at least, it is unnecessary. Also, and perhaps more significantly, a Wiggins-style approach to the distinction between de re and de dicto modality not only dissolves Fine's puzzle, but can be provided with a formal semantics that secures some of the philosophical motivations of that approach. These include reconciling true statements of de re necessity about concrete objects with the denial that there is a sense 
of 'necessarily' in which the statement 'whatever exists exists necessarily' is true.

\section{Acknowledgements}

We are grateful to Brian Ball, Derek Ball and Kit Fine for comments on a presentation of a version of this article at ENFA4, the Fourth Meeting of the Portuguese Society for Analytic Philosophy at the University of Évora in September 2009, and to David Bates, Barry Dainton, Richard Gaskin, David Price and Demian Whiting for comments on a presentation in Liverpool in February 2010. For comments on written versions, we are very grateful to Barry Dainton, Giovani Godoy Felice, Kit Fine, Mary Leng, the late E.J. Lowe, Maja Malec, Tuomas Tahko and various anonymous referees. (In most cases, the comments were on much earlier versions.)

\section{References}

Efird, David. 2010. "Is Timothy Williamson a Necessary Existent?" In Modality: Metaphysics, Logic and Epistemology, edited by Bob Hale and Aviv Hoffman, 97-107. Oxford: Oxford University Press. https://doi.org/10.1093/acprof:oso/9780199565818.003.0006.

Fine, Kit. 2005. "Necessity and Non-Existence." In Modality and Tense, 321-54. Oxford: Oxford University Press. https://doi.org/10.1093/0199278709.003.0010.

Fitting, Melvin and Richard L. Mendelsohn. 1998. First-Order Modal Logic. Dordrecht: Kluwer. https://doi.org/10.1007/978-94-011-5292-1.

Garson, James W. 2013. Modal Logic for Philosophers, 2nd ed. New York: Oxford University Press. https://doi.org/10.1017/CBO9781139342117.

Kripke, Saul A. 2011. "Identity and Necessity." In Philosophical Troubles, 1-26. Oxford: Oxford University Press. https://doi.org/10.1093/acprof:oso/9780199730155.003.0001. Originally in Identity and Individuation, edited by Milton K. Munitz, 135-64. New York: New York University, 1971.

McGinn, Colin. 2000. Logical Properties. Oxford: Oxford University Press. https://doi.org/10.1093/0199241813.001.0001.

McLeod, Stephen K. 2008. "How to Reconcile Essence with Contingent Existence." Ratio 21 (3): 314-28. https://doi.org/10.1111/j.1467-9329.2008.00404.x.

Nelson, Michael. 2016. "Contingent Existents." Philosophical Forum 47 (3-4): 361-84. https://doi.org/10.1111/phil.12128.

Prior, Arthur N. 1957. Time and Modality. Oxford: Oxford University Press.

Organon F 26 (3) 2019: 446-473 
Quine, Willard Van Orman. 1953. "Three Grades of Modal Involvement." Proceedings of the 11th International Congress of Philosophy, volume 14. Amsterdam: North-Holland. Reprinted in The Ways of Paradox and Other Essays, 156-74. New York: Random House, 1966.

Rumfitt, Ian. 2003. "Contingent Existents." Philosophy 78 (4): 461-81. https://doi.org/10.1017/S0031819103000433.

Wiggins, David. 1976. "The De Re 'Must': A Note on the Logical Forms of Essentialist Claims." In Truth and Meaning: Essays in Semantics, edited by Gareth Evans and John McDowell, 285-312. Oxford: Oxford University Press.

Wiggins, David. 1995. "The Kant-Frege-Russell View of Existence: Towards Rehabilitation of the Second Level View." In Modality, Morality and Belief: Essays in Honor of Ruth Barcan Marcus, edited by Walter Sinnott-Armstong, Diana Raffman and Nicholas Asher, 93-113. Cambridge: Cambridge University Press.

Wiggins, David. 2001. Sameness and Substance Renewed. Cambridge: Cambridge University Press. https://doi.org/10.1017/CBO9780511612756.

Wiggins, David. 2003."Existence and Contingency: A Note." Philosophy 78 (4): 483-94. https://doi.org/10.1017/S0031819103000445.

\section{Appendix: Fine on modalities}

\subsection{Grades of modality}

The purpose of this technical appendix is to further our understanding, and that of the reader, of the distinctions between modal notions that Fine makes in his article and to develop some details left aside in it. (This is despite our own scepticism about the need for, and indeed desirability of, these distinctions.) While we acknowledge that it would be useful to do this for quantified modal logic, we restrict our attention to sentential modal logic.

Recall that Fine distinguishes between restricted and extended modalities. Let ' $R$ ' be a subscript for restricted modality, ' $T$ ' be a subscript for transcendental modality, and ' $E$ ' be a subscript for extended modality. Where $A$ is any truth-valued sentence that is either purely worldly or purely unworldly, ${ }^{20}$ we shall now define restricted, transcendental, and extended notions of necessity and possibility. We take our definitions to spell out those of (Fine 2005, 3257 ), although ours differ from his in that ours do not place the same emphasis

\footnotetext{
${ }^{20}$ Such sentences contrast with the 'hybrid' sentences with which we are concerned in Section 8.2 below.
} 
on possible worlds. These definitions are not of operators in a formal language, as box and lozenge are normally taken to be, but instead are definitions of meta-linguistic predicates attached to names of sentences, after (Quine 1953): 'Nec' and 'Poss' are short for 'is necessarily true' and 'is possibly true'.

$\left(\right.$ Def $\left.\operatorname{Poss}_{R}\right) \operatorname{Poss}_{R} A=d f$ there is some worldly circumstance, $c$, such that $\mathbf{a}_{\mathbf{c}}(A)=\mathrm{T}^{21}$

$\left(\operatorname{Def~Nec}_{R}\right) \operatorname{Nec}_{R} A={ }_{d f} \operatorname{Poss}_{R} A \& \neg \operatorname{Poss}_{R} \neg A$ (i.e., for every worldly circumstance, $\left.c, \mathbf{a}_{\mathbf{c}}(A)=\mathrm{T}\right)$.

$\left(\right.$ Def $\operatorname{Nec}_{T}$ ) $\operatorname{Nec}_{T} A={ }_{d f} \mathrm{~A}$ is true $\& \neg \operatorname{Poss}_{R} A$ (i.e. $\mathrm{A}$ is true and yet there is no worldly circumstance, $c$, such that $\left.\mathbf{a}_{\mathbf{c}}(A)=\mathrm{T}\right)$.

$\left(\operatorname{Def} \operatorname{Poss}_{T}\right) \operatorname{Poss}_{T} A=d f \operatorname{Nec}_{T} A .^{22}$

$\left(\right.$ Def $\left.\operatorname{Nec}_{E}\right) \operatorname{Nec}_{E} A={ }_{d f} \operatorname{Nec}_{R} A \vee \operatorname{Nec}_{T} A$.

$\left(\right.$ Def $\left.\operatorname{Poss}_{E}\right) \operatorname{Poss}_{E} A=d f \operatorname{Poss}_{R} A \vee \operatorname{Poss}_{T} A$.

\subsection{Super-extended modalities}

(Fine 2005, 326) asserts that the idea of super-extended truth (SE-truth) (and so that of super-extended necessity (SE-necessity)) "applies to "hybrid" propositions. These are propositions ... that are composed of both worldly and unworldly components. Their truth-value in a given world turns partly on the worldly facts and also partly on the transcendental facts' ${ }^{23}$

Fine also allows for (i) hybrid predicates, i.e. predicates containing at least one worldly part and at least one unworldly part, e.g. 'is existent and is a man'; cf. (Fine 2005, 338), and (ii) hybrid terms, i.e. terms containing at least

\footnotetext{
${ }^{21}$ The restriction to worldly circumstances is necessary so as to rule out transcendental truths.

${ }^{22}$ The notions of transcendental truth, transcendental necessity and transcendental possibility are coextensive.

${ }^{23}$ As may already be evident, we prefer to work with the idea of the sentence rather than that of the proposition.
}

Organon F 26 (3) 2019: 446-473 
one worldly part that is semantically significant and at least one unworldly part that is semantically significant, e.g. 'the singleton set of Socrates'. ${ }^{24}$

Despite his allowing for these two categories, we shall presume that, for Fine, any hybrid sentence must be truth-functionally complex, i.e. that there are no atomic hybrid sentences. This is for four reasons. First, it is simpler to analyse logically complex predicates in a truth-functional way, i.e. it is simpler to analyse 'Socrates is existent and is a man' (for example) not as an atomic sentence featuring the single predication of a conjunctive predicate, but rather as a conjunction of two sentences each featuring a single predicate predicated of an identical subject, thus 'Socrates is existent and Socrates is a man'. Secondly, Fine holds that compound predicates in which one element qualifies the other are not hybrid, thus 'existent man' (for example) is a worldly predicate, not a hybrid one (Fine 2005, 337). Thirdly, Fine holds that sentences containing a hybrid name are not on that account hybrid sentences: their status depends on the status of the predicates that they contain; thus, "the singleton of Socrates is self-identical', for example, is transcendentally true. Fourthly, for Fine, a sentence with a worldly term and an unworldly predicate, e.g. (assuming that 'Socrates' is a worldly term) 'Socrates is self-identical', is unworldly, and a sentence with an unworldly term and a worldly predicate, e.g. 'the number three is being thought about by Socrates', is worldly.

Now let $A$ be any compound sentence in a truth-functional formal language, or in a truth-functional fragment of a natural language. We now define what it is to be a hybrid sentence of such a language.

Case i. Where $\zeta$ is a dyadic operator and $A$ is a sentence of the form $B$ $\zeta C, A$ is a hybrid sentence if and only if one of the following obtains:

(i) either $B$ or $C$ is a hybrid sentence;

(ii) $B$ is a worldly sentence and $C$ an unworldly sentence, or vice versa.

Case ii. Where $A$ is of the form $\neg B, A$ is a hybrid sentence if and only if $B$ is a hybrid sentence.

This concludes our definition of hybrid sentence. Let us now set out a principle that we shall then use to set up an inductive definition of the super-extended

\footnotetext{
${ }^{24}$ (Fine 2005) does not actually discuss hybrid terms, but does discuss hybrid objects using the singleton of Socrates as an example (Fine 2005, 352).
} 
modalities (SE-modalities).

(Main Principle)(MP) If $A$ is extendedly necessary (E-necessary) then $A$ is super-extendedly necessary (SE-necessary).

If $A$ is atomic then (MP) is the only way in which $A$ can be SE-necessary, since no hybrid sentence is atomic. Since $A$ is E-necessary if and only if $\neg A$ is extendedly impossible (E-impossible), and $A$ is SE-necessary if and only if $\neg A$ is super-extendedly impossible (SE-impossible), (MP) entails:

(Supplementary Principle)(SP) If $A$ is E-impossible then $A$ is SE-impossible.

If $A$ is atomic then (SP) is the only way in which $A$ can be SE-impossible, since no hybrid sentence is atomic.

(SE-equivalence) (SE) If two contingent (worldly) sentences have the same truth-value as each other across possible worlds then they are SE-equivalent; if two worldly or transcendent sentences are both E-necessary then they are SE-equivalent, and if two worldly or transcendent sentences are both E-impossible then they are SEequivalent. No other two sentences are SE-equivalent.

We also need to make use of the following theorem, which deploys the notion of SE-equivalence:

Substitution Theorem Let $A(B)$ be a sentence containing an occurrence of $B . A(B)$ is then SE-equivalent to $(B \& A(C)) \vee(\neg B$ $\& A(C))$ where $C$ is SE-equivalent to $B$.

In other words, take $A(B)$. Find a sentence, $C$, that is SE-equivalent to $B$ and substitute it for $B$. Conjoin this new sentence with $B$. This gives $B \& A(C)$ (call this 'sentence 1'). Then negate $B$ to get $\neg B$. Conjoin the result with $A(C)$. This gives $\neg B \& A(C)$ (call this 'sentence 2 '). Now disjoin sentence 1 with sentence 2 to get $(B \& A(C)) \vee(\neg B \& A(C))$. This can easily be seen to be equivalent to $A(B)$. (Proof: suppose (i) that both $A(B)$ and $B$ are true, then the first disjunct, sentence 1, will be true. Suppose (ii) that $A(B)$ is true and $B$ is false, then the second disjunct, sentence 2, will be true. Suppose (iii)

Organon F 26 (3) 2019: 446-473 
that $A(B)$ is false and $B$ is true, then both disjuncts, sentence 1 and sentence 2 , will be false. Suppose (iv) that both $A(B)$ and $B$ are false, then again both disjuncts, sentence 1 and sentence 2 , will be false. $)^{25}$

We know that every hybrid sentence contains as a proper part at least one unworldly sentence. These unworldly sentences will play the role of $B$ above. We make continued use of the substitution theorem until every embedded unworldly sentence in $A$ has been removed and replaced with an SE-equivalent worldly sentence (e.g. 'Socrates exists or does not exist' if the unworldly sentence is transcendentally true, and 'Socrates exists and does not exist' if the unworldly sentence is transcendentally false) - these sentences will play the role of $C$ above.

This means that we are left with a (possibly embedded) sentence of the form $(B \& A(C)) \vee(\neg B \& A(C))$ in which $B$ is unworldly and $C$ is a worldly equivalent of $B$. It remains for us to define when this sentence, $(B \& A(C)) \vee$ $(\neg B$ \& $A(C))$, is SE-necessary.

Suppose (i) that $B$ is transcendentally true. It follows that $C$ is a worldly necessity. It further follows, by (MP) and (SP) above, that $B$ and $C$ are SEnecessary. We now need to evaluate each of the disjuncts of $(B \& A(C)) \vee(\neg B$ $\& A(C))$. Let us start with the first disjunct. Since $A(C)$ is not a hybrid sentence - all of its component sentences are purely worldly - we already know, by (MP) and (SP) above, the modal status of $A(C)$ : if $A(C)$ is E-necessary then it is SE-necessary, if it is E-impossible then it is SE-impossible, and if it is E-contingent then it is SE-contingent. Since $B$ is SE-necessary, and a conjunction is SE-necessary if and only if each conjunct is SE-necessary (since, by its truth-table, a conjunction is true when and only when both its conjuncts are true), it follows that if $A(C)$ is E-necessary then $B \& A(C)$ is SE-necessary, if $A(C)$ is E-impossible then $B \& A(C)$ is SE-impossible, and if $A(C)$ is E-contingent then $B \& A(C)$ is $\mathrm{SE}$-contingent. But since the second disjunct is SE-impossible (since $\neg B$ is SE-impossible), irrespective of $A(C)$ ), it follows, further, that if $A(C)$ is E-necessary then $B \& A(C) \vee(\neg B \& A(C))$ is SE-necessary, if $A(C)$ is E-impossible then $(B \& A(C)) \vee(\neg B \& A(C))$ is SE-impossible, and if $A(C)$ is E-contingent then $(B \& A(C)) \vee(\neg B \& A(C))$ is SE-contingent.

Suppose (ii) that $B$ is transcendentally false. It follows that $C$ is a worldly impossibility. It further follows, by (MP) and (SP) above, that $B$ and $C$ are

\footnotetext{
${ }^{25}$ We are very grateful to Kit Fine for drawing our attention to this.
}

Organon F 26 (3) 2019: 446-473 
SE-impossible. We need, again, to evaluate each of the disjuncts of ( $B \&$ $A(C)) \vee(\neg B \& A(C))$. Let us start with the second disjunct. Again, by (MP) and (SP) above, we already know the modal status of $A(C)$, since it is not a hybrid: if $A(C)$ is E-necessary then it is SE-necessary, if it is E-impossible then it is SE-impossible, and if it is E-contingent then it is SE-contingent. But, further, since $\neg B$ is transcendentally true (since $B$ was transcendentally false), it follows that if $A(C)$ is E-necessary then $\neg B \& A(C)$ is SE-necessary, if $A(C)$ is E-impossible then $\neg B \& A(C)$ is SE-impossible, and if $A(C)$ is E-contingent then $\neg B \& A(C)$ is SE-contingent. But since the first disjunct is SE-impossible, since $B$ is SE-impossible (and a conjunction is SE-impossible if and only if at least one conjunct is SE-impossible (since, by its truth-table, a conjunction is false when and only when at least one conjunct is false)), it follows that if $A(C)$ is E-necessary then $(B \& A(C)) \vee(\neg B \& A(C))$ is SE-necessary, if $A(C)$ is E-impossible then $(B \& A(C)) \vee(\neg B \& A(C))$ is SE-impossible, and if $A(C)$ is E-contingent then $(B \& A(C)) \vee(\neg B \& A(C))$ is SE-contingent.

It may be that $(B \& A(C)) \vee(\neg B \& A(C))$ is itself an embedded sentence, in which case we just repeat the procedure now that we know the modal status of $(B \& A(C)) \vee(\neg B \& A(C))$. Since every compound sentence will be covered by repeated applications of this procedure, and since no atomic sentence is a hybrid sentence, this concludes our recursive definition of SE-modality. 